\title{
On the Performance of Soft LLR-based Decoding in Time-Frequency Interleaved Coded GFDM Systems
}

\author{
Francesco Linsalata ${ }^{1}$, Maurizio Magarini ${ }^{1}$ \\ ${ }^{1}$ Dipartimento di Elettronica, Informazione e Bioingegneria, \\ Politecnico di Milano, Italy \\ Email: name.surname@polimi.it
}

\begin{abstract}
Generalized Frequency-Division Multiplexing (GFDM) is a candidate modulation for future wireless cellular networks. The main reason stands in the flexibility of its structure that could fulfill ideas and challenges of forthcoming network scenarios. A known issue of GFDM is its worse performance compared to Orthogonal Frequency-Division Multiplexing, which is due to the interference among transmitted symbols. A mathematical model of such an interference has been proposed in a recent paper by exploiting the parallelism that exists between GFDM and discrete Gabor transform. The model allows for the design of different types of linear and non-linear equalizers. With the goal of increasing the transmission reliability, in this paper the introduction of channel coding is considered together with an appropriate interleaving. The computation of the Log-Likelihood Ratio (LLR) is described, which allows for soft decoding in the case of maximum likelihood and linear minimum mean square detection. The gain in performance achieved with channel coding and time-frequency interleaving is demonstrated by means of Monte Carlo simulations for the standard 64-state rate- $1 / 2$ convolutional code. A comparison with an approach for soft decoding proposed in literature for GFDM is also reported.
\end{abstract}

Index terms- GFDM, detection, channel coding, soft decoding, time-frequency interleaving

\section{INTRODUCTION}

In upcoming fifth generation (5G) communications, a new dawn seems to have arrived. This has an impact on all the layers of the system that must be adapted to face with the forthcoming needs, including the introduction of novel and more flexible waveforms at physical layer [1]. Among the new waveforms, Generalized Frequency Division Multiplexing (GFDM) is a flexible multicarrier modulation scheme proposed for the 5G wireless cellular systems [2]-[5].

\section{A. State of art GFDM}

In GFDM each data block is spread over different subcarriers, each of which is suitably pulse shaped by a circular filter [2]-[5]. GFDM can flexibly configure the symbol structure, since it encompasses the characteristics of both single carrier and multicarrier transmission. Moreover, low PAPR and high spectral efficiency can be achieved simultaneously by applying appropriate pulse shaping and by changing the parameters of the system [6]-[8].

However, when transmission takes place over frequency selective fading channels a performance degradation is experienced due to Inter-Symbol Interference (ISI) that arises among transmitted symbols. Therefore, suitable countermeasures for ISI must be devised [9]. Equalization, together with the introduction of channel coding, allows to reduce the detrimental effects of ISI. Thus, an appropriate modelling of ISI in GFDM is required for an optimal design of linear and non-linear equalizers. The authors of [10] were the first to propose an approximate modelling of ISI in the frequencydomain by exploiting the equivalence between GFDM and Discrete Gabor Transform (DGT) described in [11]. In [9] the new concept of Inter Sub-Symbols Interference (ISSI) among the symbols transmitted on the same sub-carrier was introduced and described in an analytical way. The developed mathematical model is based on the description of the channel associated with each sub-carrier as a Multiple-Input MultipleOutput (MIMO) system.

\section{B. Contributions}

Channel coding and interleaving are known techniques that allow for mitigation of fading effects of the channel and achieve a performance gain. Although their application to GFDM can be expected in practice, in the literature it is not yet present a study on how they can be applied to such a system. Starting from this consideration, and exploring this research aspect, the novel aspects of this work are:

- evaluation of performance of coded GFDM (CO-GFDM);

- definition of a suitable time-frequency interleaving symbol mapping for transmitted symbols;

- derivation of analytical expression for the computation of log-likelihood ratio (LLR).

We evaluate the performance of soft-decision decoding based on the interpretation of GFDM in the DGT setting and the modelling of ISSI given in [9]. A standard 64-state rate$1 / 2$ convolutional code with polynomial generator $[133,171]$ is used and the mapping of its coded bit output on the different sub-carriers is discussed [12]. A soft-input Viterbi Algorithm (VA) is used to estimate the sequence of transmitted information bits. This work paves the way to other coding and decoding scheme for interleaved CO-GFDM, which also includes iterative approaches.

\section{Main Achievements}

Our results show that CO-GFDM allows for a significant improvement in performance, thus representing a suitable approach to reduce the effect of the frequency-selectivity 
of the channel. Numerical results are provided for linear Minimum Mean-Squared Error (MMSE) equalizer and the Maximum-Likelihood Detector (MLD). It is shown that while at low-to-intermediate Signal-to-Noise Ratio (SNR) uncoded MMSE and MLD have similar Bit-Error Rate (BER), with soft decision-decoding MLD allows for superior performance compared to MMSE and other methods in the literature. Obviously, this is achieved for a higher computational cost.

The paper is organized as follows. Section II gives an overview of GFDM and its DGT interpretation. The proposed time frequency mapping for a GFDM system is introduced in Sec. III, while Sec. IV reports the soft output demodulation and LLR computation. Section V presents simulation results obtained with the proposed designs. Conclusions of this work are drawn in Sec. VI.

\section{DGT-BASED GFDM}

In the GFDM modulator, the block of transmitted bits is applied to the input of a mapper that gives at its output an $M \times K$ data matrix $\mathbf{X}$, whose $N=M K$ elements take values from a complex constellation [11]. The data matrix $\mathbf{X}$ is sent to the modulator, based on $K$ sub-carriers, where on each sub-carrier $M$ sub-symbols are transmitted. According to this model the data matrix $\mathbf{X}$ can be represented as the composition of $K$ column vectors as

$$
\mathbf{X}=\left[\mathbf{X}_{0}, \mathbf{X}_{1}, \cdots, \mathbf{X}_{K-1}\right],
$$

where

$$
\mathbf{X}_{q}=\left[X_{q}(0), X_{q}(1), \cdots, X_{q}(M-1)\right]^{T},
$$

with $X_{q}(m)$ representing the $m$ th sub-symbol, $m=0,1, \ldots$, $M-1$, transmitted on the $q$ th sub-carrier, $q=0,1, \ldots, K-1$ and $(\cdot)^{T}$ denoting the transposition operation.

After the pulse shape filtering, the $n$th sample of the transmitted GFDM signal is

$$
x(n)=\sum_{q=0}^{K-1} \sum_{m=0}^{M-1} x_{q}(m) g\left[\langle n-m K\rangle_{N}\right] e^{\frac{j 2 \pi q n}{K}},
$$

where $n=0,1, \cdots, N-1$, is the sampling index and $\langle\cdot\rangle_{N}$ denotes the modulo $N$ operation that implements the circular shifting of the periodic discrete-time shaping filter $g[\cdot]$. According to [11], (3) can be seen as the Inverse Discrete Gabor Transforms (IDGT) of the discrete-time signal transmitted $x(n)$, and $g(n)$ defines the synthesis function. In [9] the discrete-time sinc function is chosen for $g(n)$.

By following this interpretation, the receiver computes the DGT of the received signal as

$$
\hat{x}_{q}(m)=\sum_{n=0}^{N-1} \gamma^{*}\left[\langle n-m K\rangle_{N}\right] e^{\frac{j 2 \pi q n}{K}} y(n),
$$

where $\hat{x}_{q}(m)$ is an estimate of the transmitted symbols; $y(n)=$ $x(n) \otimes h(n)+w(n)$ is the received sequence after discretetime convolution with the impulse-response of the channel $h(n)$ and overlaid by the white noise $w(n) ; \gamma(n)$ is a periodic discrete function of period $N$ that, in the DGT setting, is

\begin{tabular}{|c|c|c|c|c|}
\hline$X_{0}(0)$ & $X_{0}(1)$ & $X_{0}(2)$ & $\ldots$ & $X_{0}(M-1)$ \\
\hline$X_{1}(0)$ & $X_{1}(1)$ & $X_{1}(2)$ & $\ldots$ & $X_{1}(M-1)$ \\
\hline$X_{2}(0)$ & $X_{2}(1)$ & $X_{2}(2)$ & $\ldots$ & $X_{2}(M-1)$ \\
\hline$\vdots$ & & & $\ldots$ & $\vdots$ \\
\hline$X_{K-1}(0)$ & $X_{K-1}(1)$ & $X_{K-1}(2)$ & $\ldots$ & $X_{K-1}(M-1)$ \\
\hline
\end{tabular}

Fig. 1: Encoded symbols enter the interleaving matrix by columns, i.e. following the vertical green arrow. The GFDM modulator reads the matrix by row, i.e. following the horizontal yellow arrow.

defined as the analysis function. An orthogonal transmission is achieved when $\gamma(n)$ and $g(n)$ satisfy the Wexler-Raz identity defined in [10]. This condition is automatically satisfied in case of transmission over an ideal channel, i.e. $h(n)=\delta_{n}$ being $\delta_{n}$ the Kronecher delta function $\left(\delta_{0}=1, \delta_{n}=0\right.$ for $\left.n \neq 0\right)$. For this case, in the absence of noise, $\bar{x}_{q}(m)$ coincides with $x_{q}(m)$ for each $(m, q)$ pair.

However, when transmission takes place over frequency selective channels, i.e. characterized by delay spread and fading effect, different types of interference arise and this bi-orthogonality condition is not more satisfied. While the inter carrier interference can be mitigated introducing a cyclic prefix, the interference among the sub-symbols within the same subcarrier needs to be defined and equalized. To overcome this issue in [9], the received signal on the $q$ th subcarrier $\mathbf{Y}_{q}=\left[Y_{q}(0), Y_{q}(1), \ldots, Y_{q}(M-1)\right]^{T}$ is modelled as the following MIMO system

$$
\mathbf{Y}_{q}=\overline{\mathbf{H}}_{q M} \mathbf{X}_{q}+\mathbf{W}_{q}, \quad q=1, \ldots, K-1,
$$

where $\mathbf{W}_{q}=\left[W_{q}(0), W_{q}(1), \ldots, W_{q}(M-1)\right]^{T}$ is the DGT vector of the noise samples and

$$
\overline{\mathbf{H}}_{q M}=\left[\begin{array}{cccc}
\bar{H}_{q M}(0) & \bar{H}_{q M}(N-K) & \ldots & \bar{H}_{q M}(N-(M-1) K) \\
\bar{H}_{q M}(N-(M-1) K) & \bar{H}_{q M}(0) & \cdots & \bar{H}_{q M}(N-K) \\
\vdots & \vdots & \ddots & \vdots \\
\bar{H}_{q M}(N-K) & \bar{H}_{q M}(N-2 K) & \cdots & \bar{H}_{q M}(0)
\end{array}\right]
$$

describes the impact of the channel matrix on the the transmitted vector. As discussed in [9], it is possible to apply different types of per sub-carrier MIMO equalization approaches to deal with ISSI and symbols detection. However, it is worth noting that in [9] the analysis of the performance is limited to uncoded transmission of the information bits.

\section{TIME-FREQUENCy INTERLEAVING IN GFDM SETTING}

Starting from the representation of the data matrix given in (1) and considering the received signal vector defined in (5) the aspect of time-frequency interleaving is here considered. The encoded bits are interleaved over the $K$ subcarriers as shown in Fig. 1. This counteracts the frequency selectivity of the channel and increases the diversity, thus 


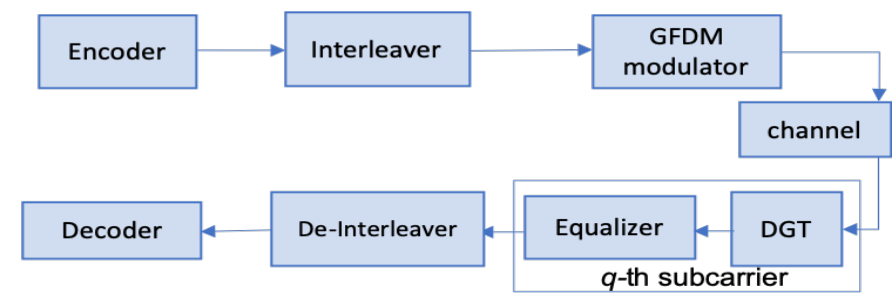

Fig. 2: DGT-based Interleaved Coded-GFDM

improving the performance. In fact, in the adopted timefrequency interleaving $K$ symbols are firstly distributed across different subcarriers with the aim of combing the diversity effect of the channel frequency selectivity on the $K$ subcarriers, averaging the "bad" sub-carriers with the "good" ones. The term "bad" associated with a given sub-carrier means a sub-channel characterized by a deep-fading effect, which makes the channel condition inadequate for a reliable transmission. Then, the successive blocks of $K$ symbols fill the $M-1$ available time slots. It is worth observing that codewords sufficiently long provide a sort of averaging effect with respect to fading events since they introduce logical relations between bits subject to different channel conditions. Another advantage of this approach is the reduction of the system complexity. In fact, a single encoder and decoder can be used in the entire system as shown in Fig. 2 .

\section{Soft-Output Demodulation And LLR COMPUTATION FOR DGT-BASED GFDM}

The received sequence is demodulated by a soft-input softoutput demodulator. The computed LLR is then passed to a standard binary soft-input Viterbi decoder. The soft decoder uses the sequence of LLR values to take a decision on the transmitted information bits. The LLR analysis is described in the following subsections, taking into account the different types of receivers and the discussed time-frequency mapping.

\section{A. Linear MMSE Equalizer}

The linear MMSE equalizer allows for good trade-off between noise enhancement and ISI removal [14]. The expression of the demodulation matrix for the $q$ th subcarrier, $q=0, \ldots, K-1$, is

$$
\mathbf{C}_{q M, \mathrm{MMSE}}=\left(\overline{\mathbf{H}}_{q M}^{H} \overline{\mathbf{H}}_{q M}+N_{0} \mathbf{I}_{M}\right)^{-1} \overline{\mathbf{H}}_{q M}^{H},
$$

where $N_{0}$ is the variance of the noise and $\mathbf{I}_{M}$ is an $M \times M$ identity matrix. After the equalization step, an estimate of the transmitted symbols on the $q$ th subcarrier $\overline{\mathbf{X}}_{q}=\mathbf{C}_{q M, \text { MMSE }} \overline{\mathbf{Y}}_{q}$ is passed to the demodulator. Since the modulation procedure is memoryless, the demodulation of $\overline{\mathbf{X}}_{q}$ can be decomposed into independent demodulations of its components. The soft de-mapper calculates the LLR metric for the coded bit associated with it. Assuming Quadrature Phase Shift Key (QPSK) modulation, i.e. two bits $c_{q, m, 1}$ and $c_{q, m, 2}$ per symbol $X_{q m}$, the LLR for coded bit $c_{q, m, 1}$ is

$$
z_{c_{q, m, 1}}=\ln \frac{\operatorname{Pr}\left(c_{q, m, 1}=1 \mid \bar{X}_{q m}\right)}{\operatorname{Pr}\left(c_{q, m, 1}=0 \mid \bar{X}_{q m}\right)}, \quad \begin{array}{r}
q=0, \ldots, K-1, \\
m=0, \ldots, M-1 .
\end{array}
$$

where $\ln (\cdot)$ denotes the natural logarithm and $\operatorname{Pr}\left(c_{q, m, 1}=\right.$ $\left.b \mid \bar{X}_{q m}\right)$, with $b=0,1$, is the a priori conditional probability that $c_{q, m, 1}=b$ given that the estimated symbol is $\bar{X}_{q m}$ and can be rewritten as

$$
\operatorname{Pr}\left(c_{q, m, 1}=1 \mid \bar{X}_{q m}\right)=\sum_{c_{q, m, 2}=0}^{1} \operatorname{Pr}\left(c_{q, m, 1}=1, c_{q, m, 2} \mid \bar{X}_{q m}\right)
$$

By using the Bayes' rule and assuming equiprobable transmitted and received symbols, (8) becomes

$$
z_{c_{q, m, 1}}=\ln \frac{\sum_{c_{q, m, 2}=0}^{1} f\left(\bar{X}_{q m} \mid c_{q, m, 1}=1, c_{q, m, 2}\right)}{\sum_{c_{q, m, 2}=0}^{1} f\left(\bar{X}_{q m} \mid c_{q, m, 1}=0, c_{q, m, 1}\right)} .
$$

Now, since it can be assumed that, after the linear equalization, the components of the transmitted codeword undergo independent channel fading gain and noise samples, the conditional Probability Density Function (PDF) follows a Gaussian distribution as

$$
f\left(\bar{X}_{q m} \mid X_{q m}\right)=\frac{1}{\sigma_{m} \sqrt{\pi}} e^{-\frac{\left(\bar{X}_{q m}-X_{q m}\right)^{2}}{\sigma_{m}^{2}}}
$$

where $\sigma_{m}^{2}=N_{0}\left(\left(\mathbf{H}_{q M}^{H} \mathbf{H}_{q M}+N_{0} \mathbf{I}_{M}\right)^{-1}\right)_{m, m}$ is the noise variance after the equalizer. Substituting (11) in (10) and making all the derivations also for $c_{q, m, 1}=0$ case in the denominator of (8), the value of the LLR for $c_{q, m, 1}$ is computed. Note that for $c_{q, m, 2}$ the same derivation done for $c_{q, m, 1}$ in the previous equations holds by inverting 1 with 2 in the subscripts of the variables, i.e. considering the previous coded bit $c_{q, m, 1}$ in the conditional probability (9). Moreover, in case of high order modulation formats with $l$ bits per symbol, (9) considers the sum of all the possible $l-1$ bits combinations $\mathbf{c}_{l-1}$ as

$$
\operatorname{Pr}\left(c_{q, m, 1}=1 \mid \bar{X}_{q m}\right)=\sum_{\mathbf{c}_{l-1} \in \mathcal{B}^{l-1}} \operatorname{Pr}\left(c_{q, m, 1}=1, \mathbf{c}_{l-1} \mid \bar{X}_{q m}\right),
$$

where $\mathcal{B}^{l-1}$ is the set defined by the $(l-1)$-dimensional Cartesian product of $\mathcal{B}=\{0,1\}$.

\section{B. $M L D$}

In the case of MLD, the approach followed to estimate the LLR is based on an exhaustive search per detection [15], [16]. Considering again Gray mapped-QPSK, we report how the demodulation is done for the first symbol, i.e. $m=1$ in the subscripts, received on the $q$ th sub-carrier $\bar{Y}_{q 1}$ as explained in the following. The symbol $\bar{Y}_{q 1}$ is characterized by two coded bits $c_{q, 1,1}$ and $c_{q, 1,2}$. The LLR is defined as

$$
z_{c_{q, 1,1}}=\ln \frac{\operatorname{Pr}\left(c_{q, 1,1}=1 \mid \overline{\mathbf{Y}}_{q}\right)}{\operatorname{Pr}\left(c_{q, 1,1}=0 \mid \overline{\mathbf{Y}}_{q}\right)} \quad q=0, \ldots, K-1 .
$$


The conditional probability of $c_{q, 1,1}=1$ given the received symbols $\mathbf{Y}_{q}, \operatorname{Pr}\left(c_{q, 1,1}=1 \mid \overline{\mathbf{Y}}_{q}\right)$ can be rewritten as

$$
\begin{gathered}
\operatorname{Pr}\left(c_{q, 1,1}=1 \mid \overline{\mathbf{Y}}_{q}\right)=\sum_{c_{q, 1,2}=0}^{1} \operatorname{Pr}\left(c_{q, 1,1}=1, c_{q, 1,2} \mid \overline{\mathbf{Y}}_{q}\right)= \\
=\operatorname{Pr}\left(a_{q, 1}=-1 \mid \overline{\mathbf{Y}}_{q}\right)+\operatorname{Pr}\left(a_{q, 1}=-j \mid \overline{\mathbf{Y}}_{q}\right)= \\
=\sum_{\mathbf{a}_{M-1} \in \mathcal{A}^{M-1}} \operatorname{Pr}\left(a_{q, 1}=-1, \mathbf{a}_{M-1} \mid \overline{\mathbf{Y}}_{q}\right)+ \\
\quad+\sum_{\mathbf{a}_{M-1} \in \mathcal{A}^{M-1}} \operatorname{Pr}\left(a_{q, 1}=-j, \mathbf{a}_{M-1} \mid \overline{\mathbf{Y}}_{q}\right)
\end{gathered}
$$

where $\mathcal{A}^{M-1}$ is the set defined by the $(M-1)$-dimensional Cartesian product of $\mathcal{A}=\{1,-1,-j, j\}$ and $\mathbf{a}_{M-1}$ is the vector of one possible combination. For the Bayes rule, using (11) we get

$$
\sum_{\mathbf{a}_{M-1} \in \mathcal{A}^{M-1}} \operatorname{Pr}\left(a_{q, 1}=-1, \mathbf{a}_{M-1} \mid \overline{\mathbf{Y}}_{q}\right)=\sum e_{\mathbf{a}_{M-1} \in \mathcal{A}^{M-1}} e^{\frac{-\left\|\overline{\mathbf{Y}}_{q}-\overline{\mathbf{H}}_{q} \mathbf{a}_{M-1}\right\|^{2}}{N_{0}}} .
$$

Note that taking the $\max _{\mathbf{a}_{M-1} \in \mathcal{A}^{M-1}} \sum_{\mathbf{a}_{M-1} \in \mathcal{A}} e^{\frac{-\left\|\overline{\mathbf{Y}}_{q}-\overline{\mathbf{H}}_{q} \mathbf{a}_{M-1}\right\|^{2}}{N_{0}}}$, i.e. the combination of the $M-1$ symbols that maximizes it, the MLD condition [16] is obtained. In the same way, the other term in the second line of (14) can be rewritten as

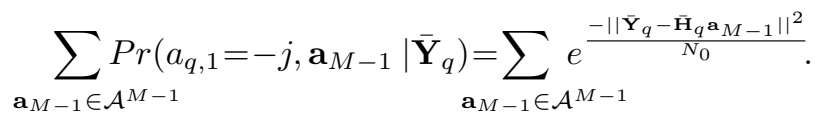

By following the same consideration for $\operatorname{Pr}\left(c_{q, 1,1}=0 \mid \overline{\mathbf{Y}}_{q}\right)$, the LLR is obtained, and so on for all the other bits. As it can be noted, when the constellation size increases, this approach leads soon to unaffordable complexity that rises exponentially. Then, these metrics are de-interleaved, i.e. each couple is assigned to the bit position in the decoded sequence according to the de-interleaver map and fed to the decoder. To perfom the decoding we consider soft-input VA with unquantized LLR values [17]. The VA selects the binary sequence with the smallest cumulative sum of metrics [15].

\section{Simulation Results}

Here, we report a comparison between all the different types of receivers discussed in the previous section, including that proposed in [10]. This latter, named approximated MLD, implements an independent frequency-domain computation of the LLR for the sub-symbols transmitted on the same subcarrier by neglecting the interference generated by the other remaining $M-1$ sub-symbols.

Taking into account the $L$-path tapped delay line channel model defined in [13], the time impulse response of the multipath fading channel results to be as

$$
h(n)=\sum_{i=0}^{L-1} h_{i} \delta\left(n-\tau_{i}\right),
$$

where $h_{i}$ is tap coefficient of the $i$ th path, with random complex amplitude following Rayleigh distribution, associated with the propagation delay $\tau_{i}$ and $\delta(t)$ is the delta Dirac function. In all the simulations $L=9$ is used. The propagation delays in (17) are equally spaced, i.e. the case where $\tau_{i}=i$, with $i=0, \ldots, L-1$. This approach can be generalized in case of not uniform delays using an interpolation and over fitting procedure that takes into account the sampling intervals $T=\frac{1}{N \Delta f}$, where $\Delta f$ is the subcarrier spacing, to translate the associated channel power in the correct $i$ position. This type of channel model includes also the 9-paths EVA channel channel used in [10] and defined in the 3GPP LTE standards.

Figure 3 reports the BER versus the ratio between the energy per bit and noise variance $E_{b} / N_{0}$ of the considered encoding scheme with respect to uncoded transmission. It can be observed that, notwithstanding the difference in complexity, MMSE and MLD receivers exhibit similar performance for the entire range of considered SNR value in case of uncoded transmission. In contrast, for coded transmission the MLD allows for better performance compared to linear MMSE. However, both of them outperform the approximate MLD approach [10], meaning that the exploited ISSI modelling allows a better design of the equalizers.

In Fig. 4 it is reported the BER performance of GFDM for QPSK transmission with different values of $M$ and $K$, i.e. by varying the number of sub-carriers involved in the transmission $K$ for a fixed number of symbols per sub-carrier $M$ or, by keeping $M$ fixed and changing $K$. This is done to show how the time-frequency interleaving described in Sec. 3 impacts the performance. From these results some considerations can be done about the overall of $N=M K$ symbols transmission for different values of $K$ and $M$. As it can be seen, by increasing $K$ for $M=5$, a $2 \mathrm{~dB}$ performance degradation at $\mathrm{BER}=5 \cdot 10^{3}$ is observed, which is due to the higher incidence fading effects on average. Instead, by increasing $M$ for $K=32$ a slightly difference appears starting from $E_{b} / N_{0}=8 \mathrm{~dB}$, which demonstrates the diversity gain effect obtained by transmitting more sub-symbols on the same sub-carrier. Thus, for a fix $N$ : if

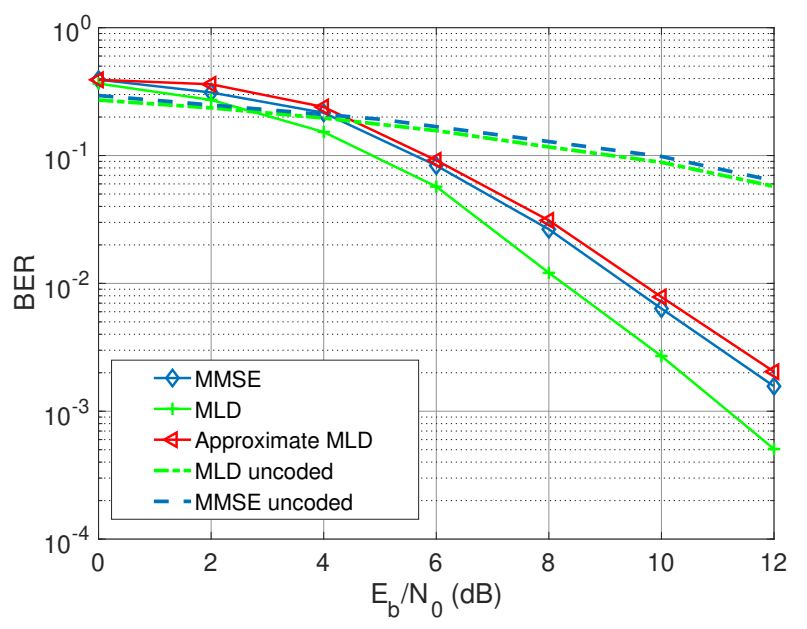

Fig. 3: CO-GFDM QPSK BER vs. $E_{b} / N_{0}$ with $M=5$ and $K=32$ and different types of per sub-carrier receiver 


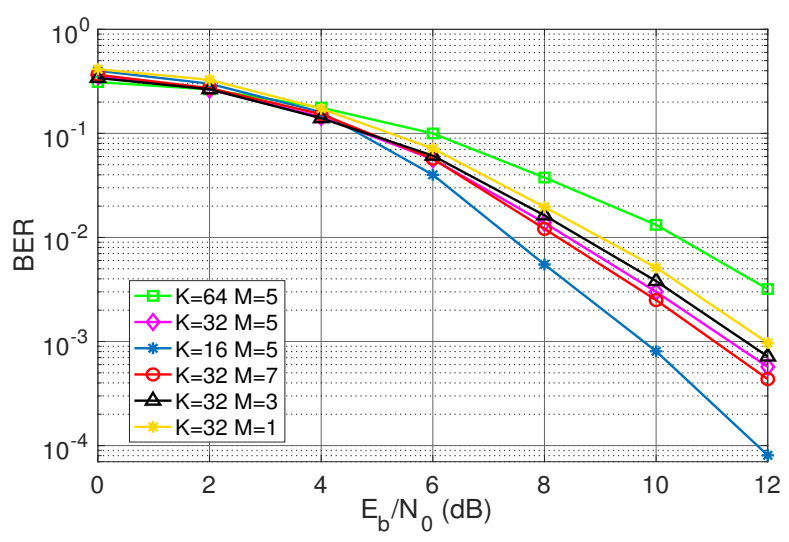

Fig. 4: CO-GFDM QPKS BER vs. $E_{b} / N_{0}$ with different number of transmitted information symbols $N$

more subcarriers $K$ are considered a performance degradation is shown due to channel selectivity, but this allows to reduce the number of sub-symbols $M$ and thus, the complexity due to ISSI equalization; while if $M$ is increased, reducing the number of sub carriers $K$, the proposed interleaving gain makes the transmission more robust to fading, allowing a performance gain at the cost of higher complexity.

Figure 5 shows the BER versus $E_{b} / N_{0}$ for higher order modulation schemes, i.e. 16-QAM. Since in this case the MLD approach turns to be unpractical from a computational cost point of view, only the performance of linear MMSE equalizer is reported. Without loss of generality and for completeness, the same simulation parameter in [Tab. IV of [10]] are investigated. A 3GPP deterministic EVA channel model profile is considered, with $K=256$ and $M=7$. It is worth noting that the difference in trend of two equalization procedures is consistent with the one of Fig. 3 .

\section{CONCLusion}

Generalized Frequency-Division Multiplexing (GFDM) is a newly proposed multi-carrier modulation scheme that can meet the requirements of flexibility and higher spectral efficiency of future high frequency cellular networks. In this paper we have considered the problem of making GFDM transmission more robust to multipath fading by introducing and modelling channel coding after an appropriate interleaving of information symbols, exploiting both the frequency and time domains. The computation of LLR is discussed and detailed for linear MMSE and MLD using a recently proposed modelling of the interference that affects symbols transmitted on the same subcarrier. The improvement in error rate performance achieved with the proposed LLR computation and mapping over another approach proposed in the literature is demonstrated by means of Monte Carlo simulations for soft-decision decoding of the standard 64-state rate-1/2 convolutional code.

Furthermore, some suggestions about the waveform design parameters, i.e. $M$ and $K$, arise from the simulation results

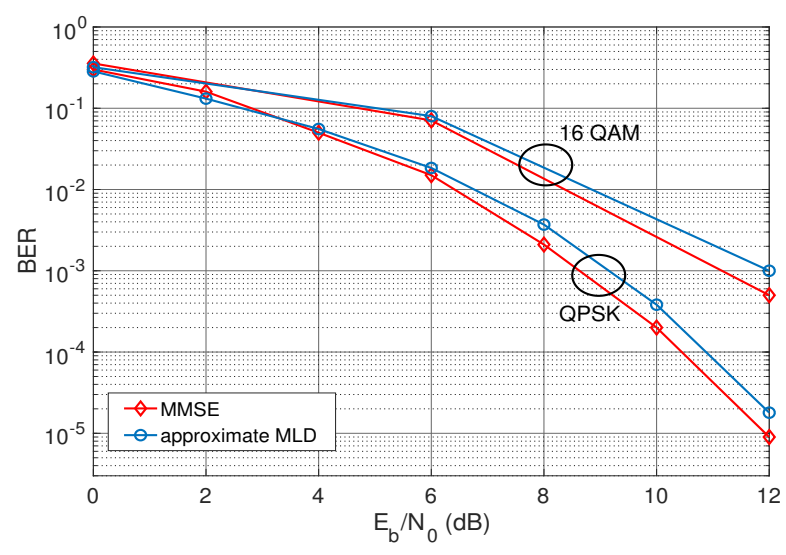

Fig. 5: CO-GFDM QPSK and 16-QAM BER vs. $E_{b} / N_{0}$ in case of 3GPP EVA channel [10] with $K=256$ and $M=7$

discussion, allowing for a trade off between performance gain and complexity.

\section{REFERENCES}

[1] H. Shimodaira et al. "Enhanced next generation millimeter-wave multicarrier system with generalized frequency division multiplexing," Int. J. Antenn. Propag., vol. 2016, 11 pages, 2016.

[2] X. Zhang et al., "On the waveform for 5G," IEEE Commun. Mag., vol. 54, no. 11, pp. 74-80, Nov. 2016.

[3] Y. Cai et al., "Modulation and multiple access for $5 \mathrm{G}$ networks," IEEE Comm. Sur. Tut., vol. 20, no. 1, pp. 629-646, Mar. 2018.

[4] A. Zaidi et al., "Waveform and numerology to support 5G services and requirements," IEEE Commun. Mag., vol. 54, pp. 90-98, Nov. 2016.

[5] N. Michailow et al., "Generalized frequency division multiplexing for 5th generation cellular networks," IEEE Trans. Comm., vol. 62, no. 9, pp. 3045-306, Sept. 2014.

[6] G. Wunder et al., "5GNOW: non-orthogonal, asynchronous waveforms for future mobile applications," IEEE Commun. Mag., vol. 52, no. 2, pp. 97-105, Feb. 2014.

[7] M. Sameen et al., "Comparison of GFDM and OFDM with respect of SER, PSD and PAPR", Int. J. Mech. Eng. Comput. Appl., vol. 4, 2016.

[8] N. Michailow and G. Fettweis, "Low peak-to-average power ratio for next generation cellular systems with generalized frequency division multiplexing," in Proc. of IEEE ISPACS, 2013, pp. 651-655.

[9] A. Kumar and M. Magarini, "On the Modeling of Inter-Sub-Symbol Interference in GFDM Transmission," in IEEE Commun. Lett., vol. 23, no. 10 , pp. 1730-1734, Oct. 2019.

[10] P. Wei et al., "Fast DGT-based receivers for GFDM in broadband channels," IEEE Trans. Commun., vol. 64, pp. 4331-4345, Oct. 2016.

[11] M. Matthé et al., "Generalized frequency division multiplexing in a Gabor transform setting," IEEE Commun. Lett., vol. 18, pp. 1379-1382, Aug. 2014.

[12] C. Lee. Convolutional coding: fundamentals and applications. Boston, MA: Artech House, 1997.

[13] M. K. Simon and M.-S. Alouini, Digital Communication over Fading Channels, 2nd ed. New York: Wiley, 2005.

[14] S. Yang and L. Hanzo, "Fifty years of MIMO detection: the road to large-scale MIMOs," IEEE Commun. Surv. Tutor., vol. 17, no. 4, pp. 1941-1988, 2015.

[15] F. Tosato, P. Bisaglia., "Simplified soft-output demapper for binary interleaved COFDM with application to HIPERLAN/2," in Proc. of ICC, 2002, vol. 5, pp. 664-668.

[16] H. Artes et al., "Efficient detection algorithms for MIMO channels: a geometrical approach to approximate ML detection," IEEE Trans. Sig. Proc., vol. 51, no. 11, pp. 2808-2820, Nov 2003.

[17] G. D. Forney, "The Viterbi algorithm," Proc. IEEE, vol. 61, pp. 268-278, Mar. 1973. 